\title{
Aus Kölner ProzeBakten. \\ Beiträge zur Geschichte der Sittenzustände in Köln im 15. und 16. Jahrhundert.
}

\author{
Von JUSTUS HASHAGEN.
}

\begin{abstract}
Das Kölner Stadtarchiv besitzt in seinen weltlichen Prozeßakten, bestehend aus noch nicht verzeichneten Protokollen, aus dem Liber Malefactorum (1510-1522) und der großen Reihe der "Turmbücher" ein unendlich reiches Material für die Sittengeschichte des 15. bis 18. Jahrhunderts. Die erstaunliche Ausführlichkeit, mit der in diesen Akten die Zeugenaussagen protokolliert worden sind, gibt ein überaus anschauliches Bild von dem sittlichen Habitus der Zeit. In den folgenden Mitteilungen ist nur das geschlechtliche Gebiet berücksichtigt worden und hier wieder nur die Summe von Prozessen, welche das gewöhnliche Maß überschreiten, ${ }^{1}$ ) indem sie von qualifizierter und widernatürlicher Unzucht handeln. Es soll damit natürlich nicht die Vorstellung erweckt werden, als wenn es berechtigt wäre, daraus Schlüsse auf eine allgemeine Verbreitung solcher Verbrechen zu ziehen. Dazu liegt kein zwingender Grund vor. Wohl aber zeigen diese protokollarisch getreuen Angaben, ähnlich wie der von mir in der Westdeutschen Zeitschrift XXIII (1904), $139 \mathrm{ff}$. veröffentlichte westfälische Bericht, daß auch in Köln, der Hochburg der kirchlichen Partei vor 1540, das Gefühl von den sittlichen Schranken gegen Ende des Mittelalters mehrfach gänzlich abhanden gekommen ist. Auch diese unvollständigen Mitteilungen aber sind wieder geeignet, die Unzulänglichkeit aller argumenta e silentio auf diesem Gebiete zu erweisen.
\end{abstract}

1) Zur Ehebruchsstatistik ergibt das Verbrecherbuch folgende Zahlen: 1516:1, $1517: 11,1518$ und 1519 je 1,1520 und 1521 je 3. 
Denn es ist ausgeschlossen, daß die sittliche Gesamthaltung in einer Stadt hoch über dem Durchschnitt steht, wenn Beispiele qualifizierter Unzucht: Unzucht mit Hausgenossinnen, schwere Notzucht-, Kuppelei- und Inzestfälle auftreten oder wenn es nötig ist, gegen homosexuelle Vergehen oder den geschlechtlichen Mißbrauch von Minderjährigen selbst von Rats wegen Schritte zu tun.

Vor einfachen Relationen haben Prozeßakten immer den Vorzug, daß vielfach die verschiedenen Parteien zu Worte kommen. Es ist also möglich, danach ein ganz genaues Bild zu entwerfen. Jedenfalls aber sind die Akten Quellengruppen, deren Glaubwürdigkeit über allem Zweifel erhaben ist.

$\mathrm{Da}$ es sich um weltliche ProzeBakten handelt, so sind Geistliche fast garnicht beteiligt. Zur Beleuchtung der Sittengeschichte des Klerus müßten die Offizialatsakten ergänzend eintreten. Schon in dem genannten Aufsatze der Westdeutschen Zeitschrift ist auf einige interessante Stücke der Kölner Bestände hingewiesen worden. Besondere Beachtung verdient auch der bei den Universitätsakten beruhende Prozeß gegen den Priester Johannes de Remelsheim (1467, Mai 25.-Sept. 7.), dessen Kenntnis ich der Güte des Herrn Dr. Keußen verdanke (vgl. Westdeutsche Zschr. XVIII, 357, Mitt. aus dem Kölner Stadtarchiv XXIV, 60, Nr. 77). Ihm und Herrn Prof. Hansen bin ich für Lesung und Interpretation der folgenden Akten zu Dank verpflichtet. Auch andere Abteilungen des Kölner Stadtarchivs enthalten wertvolles sittengeschichtliches Material.

1.

\section{Verhandlung gegen Figgin Offermanns, Frau des Kochs im Deutschen Hause, wegen Kuppelei. 1538, Nov. 4. und 7.}

Von den eirsamen heren Johan von Gereßhem und Diederich Horner, thornmeisteren, ist Catharingin, Jacob Mulners dochter, verhoirt worden, die gesacht und bekentlich gewest, dat sie verlittener zeit und uff dach, doe die leude an der mollen verdroncken synt, van Figgin, Johan Offermans dochter und des kochs frauwe im Duitzen hauß, uß des vatters hauß geholt in meynong oder under dem scheyn, sie voir den Mynrebroederen 
by eyner lynenneyerschen ${ }^{1}$ ) $\mathrm{zu}$ verhelffen, wie sie aber keyner lynenneyerschen van Figgin angeboeden. Dan sie mit sich heym genoemen und myt ein par roeder mauwen, ${ }^{2}$ ) eyn damretten ${ }^{3}$ ) cammellotten ${ }^{4}$ ) klier und weiß haltzdocher und mutzen getziert und voir sent Aperen aver sent Apostolen kloister hinder over gen wall uff der Ehelendigen ${ }^{5}$ ) kirchoiff durch eyn cleyn nydder durgin under der erden aver eyn byr-ader weynstellonge (wilch dorchgen Anna, des Compturs macht, die mit darby gewest, uffgeslossen) in dat Duitze hauß uff gemelten Comptuirs heymlich gemach gefuirt und braicht.

Dar Anna, des Compturs macht, innen die kost bracht, und so sie nu mit sampt den Comptur gessen haven, hait der Comptur und der koch, Figgins man, darumb gespilt und gedobbelt, of der Comptur by Figgin oder Catharingen slaiffen solde. Doch ist Catharingen den Comptur zu deyll gefallen.

Und so idt spaede worden ist, haiff Catharingin myt Figgin heymen willen gaen. Und so sie an der portzen koemen ist, haidt Figgin ir die portze voir der naesen zugeslaegen, und der Comptur haiff sie by eynen armen genoemen und Catharingen widder uff seyn heymlich gemach geleidt und sie bevolen, dat sie sich uBdoen solde, umb by im zu slaiffen. Haiff sie doch ein stuyffgin by des Compturs gemach gesehen, dair siè uff geloiffen ist und dat zugefirckelt. ${ }^{6}$ ) Und wie woll der Comptur sie, umb das uffzudoen, offt und duck erfoddert, haiff sie das nyt willen doen. Dan den nacht daruff verbleven.

Aber des anderen morgens haiff sie Figgin widder gehoirt, darumb solch firckell uffgedain, und so sie by Figgin kommen is und Figgin gehoirt, dat der Comptur synen willen nit geschafft hait, haiff Figgin uff Catharingin qwait ?) worden, zu Catharingen gesacht: "Du unvelaet, ${ }^{8}$ ) du wiltz noch kanst geyn guide daege haven. Krigen dich dyn alderen, sie werden uch

1) Leinennäherin.

2) Ärmel.

3) Von der Farbe des Damhirsches. Vielleicht ist hier ähnlich wie Buch Weinsberg IV, 256 dannetten (lohbraun) zu lesen.

4) Aus Camelotzeng, eigentlich Zeug aus Kamelshaaren: Buch Weinsberg a. a. O.

5) Der Fremdenkirchhof bei S. Katharina.

6) Zuriegeln.

7) Zornig.

8) Unflat. Hier Schimpfwort. 
worgen und also myßhandelen, das die $d r o i ß 1)$ und pestilencii werden uch plaegen."

Derhalven sie vermeynt gehadt, das iren alderen sulchs bewust gewest, und dem Comptuir acht daege und so lange, das der Comptur sie heym gesant haiff, zo willen gewest und haiff's von dem Comptur irstlichen genossen eynen kroinen in stadt eyns miedepennincks. Darnach haiff der Comptur Figgin gegeven druitzien gulden, umb Catharingin zu cleiden. Er vermeynt aber, der solde nyt mher dan 11 gulden gewest seyn. Und Figgin haiff Catharingin darvan gegulden eynen roden rock, eynen Arnissche ${ }^{2}$ ) hoick, ${ }^{3}$ ) eyn par worsten ${ }^{4}$ ) mouwen, eynen darmitten (!) camelotten clier. Und ist van dem gelde avergelouffen seeß marcke, die der Comptur Figgin voir unlust gegeven hait.

\section{Eodem die.}

Demnach Jacob Mulners, Catharingins vader, by seynem eyde erfraicht, sacht, Figgin Offermans haiff seyne dochter in eyme scheyne by eyne lynenneyers hinder den Mynrerbroederen $\mathrm{zu}$ brengen (!). Doch so sie nach acht daegen heym koemen und er und seyne hußfrauwe die sache ußfraicht hant und auch der gestalt der sachen, wie vurs., erinnert worden synt und die dochter gestraifft hant: hait ir dochter gesacht und innen geclaicht, dat sie vurs. maissen darzu hin koemen und all doeren ir voirgesloissen worden synt. Haeff der vaeder ir bevolen, dat sie sulchs bichten soll etc.

Doch van syner frauwen gehoirt, dat sie zu im gesacht haiff, das des Comptuirs maicht [Anna] ir eynen par hoisen und wamme $\beta$ bracht haiff und gesacht: "Gib das dynem man. Eß ist meynem man zu cleyn. Und slait das metgin nyt! Sie is nyt umbsuyß ußgewest. Sie hait geltz genoich verdient. “”

Anno vurs. 7. Nov.

Figgin leugnet alles trotz vorgenommener Konfrontation mit Catharina.

1) Drüse. Dies ist ein sehr beliebtes Schimpfwort, wie meine Zusammenstellung in der Westdeutschen Zeitschrift XXIII, 112, A. 17 zeigt.

2) Von Arras.

3) Kapuze.

4) Von Kammgarn. 
... Hant meyne heren torenmeisteren Annen van Orssenburg, itzont des prabst macht zu sent Joris, voir des Compturs macht gewest, verhoirt, die gesacht und getzucht hait:

Das ir her, der Comptuir, by sye in die koch kommen und umb tzwae personen kost uff seyne, des Compturs, gemach $\mathrm{zu}$ brengen bevolen.

So sie im die kost daruff bracht hait, haiff sie Figgin und Catharingin by iren heren sitzende uff des heren gemach vonden, wilche Catharingin mit seir lichten ${ }^{1}$ ) cleidern gecleidt gewest sy, also das dieser getzuch [Anna] zu iren heren gesacht haiff: „Daß metgin is voll luyB.2) Gyb innen eynen gulden ader tzwentzich, das es gecleidt werde." Derhalven der Comptur eynen gulden oder tzwentzig ungeferlich doen hoelen und Figgin gedain, umb Catharingin zu kleiden, wie das metgin auch gecleit worden ist. Auch getzucht, das sie haiff das metgin sehen weirden ${ }^{3}$ ) oder schreien und diesen getzuch gebeden, dat sie by ir slaiffen mocht etc. Und gesacht, dat sie anen den Ehelendigen kirchoff under der erden aver eynen stellong her inkommen synt. Und das sulchs wair ist, will sie by staen und halden.

Erst als man der Figgin droht, eine Hebamme (weyßmoder) $z u$ holen, gesteht sie alles. Sie wird gegen Urfehde und Bürgschaft aus dem Gefängnisse entlassen.

2.

\section{Verhandlung gegen Michel in der Friesenstraße wegen Unzucht und Ehebruchsversuchs mit seiner Stieftochter. [1488 - 1498.]}

Also eyn geruicht is, dat eynre genant Michell woinafftich up der Vriessenstrayssen, syne stieffdoichter bekant ind mit kynde gemacht soele haven, ind do die selve vur eyn junffer an eynen gesellen bestait ind gehylicht, synt etliche getzuige darup verhoirt worden, die selven ouch by den eyden, die sy up den Verbuntbrieff gedain, gesacht und gekundt haint, inmaissen herna geschreven steyt....

1) Wenig.

2) Laus; mnd. lûs.

3) Sich wehren. 
Der geweldtrichter vurs. [Heinrich Liblar 1488-1498] hait in bywesen der geschickter heren Kyrstgen, der vurs. personen broeder, gefraicht, off der selve nyet zo yeme komen geweist were und gesacht hedde, syn stieffader hedde syne stieffdoichter (syne suster) mit kynde gemacht ind were ouch naderhant, as syn suster an die hillige ee bestait were, zo synre suster komen und sie angesont, dat sy im synen willen mit yre weulde laissen schaffen, dat syn suster yeme allet zo doyn geweigert. Darumb der stieffader sy understanden soulde haven mit eyme metze zo slayn....

Johan Stümpgen sait, wie yeme kundich sy, dat Michell vurs. buyssen der stat up syn lant eyn cleyn huysgen gemacht hedde, as ouch eynsdeils andere up yre lant hedden laissen machen. In deme selven huysgen hedde eynre genant Peter Schüyrgen, wonafftich achter sent Apren, zo anderen tzyden den gemelten Michell und syne stieffdoichter samen sien ligen, ind hedden sich beyde samen in den arm genomen, as yeme der selve Peter muntlichen gesacht hadde....

Belastendes Zeugnis Johan Wredes. Kompensationsverhandlungen mit dem Manne der Stieftochter:

Herman, der stieffdoichter vurs. elige man, sait, wie dat Michell mit synen frunden yeme syne stieffdochter vur eyne junffer zo eynre eligen huysfrauwen gegeven ind in darome bestait have; ind as der bruloffs dach geschiet ind syn huyssfrauwe yeme zo bedde komen was, do hait sich erfunden, dat sy geyne junffer ind mit kynde gemacht was. Do have hey van yr eyn wissen haven willen, wer der vader van deme kynde were; dat sy yeme eyne tzytlanck allet verswiegen hait ind nyet sagen woulde. Ind as nu Herman zome lesten sulchs umber wissen woulde, do have syn huysfrouwe zo yeme gesproichen: weulde hey yre nyet entlouffen ind sy ouch nyet verschemen, so weulde sy it yeme sagen, ind have yeme do gesacht, wie dat yre stieffader yre dat kynt kurtz na payschen nyestleden gemacht hedde. Ind der selve were ouch naderhant tzweymaile, as sy syne eefrauwe worden was, zo yre komen ind sy angesont ind begert hedde, dat sy im synen willen mit yre weulde laissen schaffen, des sy yeme geweigert have. Deshalven yre stieffader van bedroeffnisse syne hant 
up syn metz geslagen hedde. Ind Johan Wrede sy ouch by yeme geweist ind yeme vurgehalden have, dat hey die sachen verswiegen halde ind eyne fruntschafft van gelde darvur nemen weulde. Des hey nyet en have willen doyn ind gesacht, hey weulde, dat yeme eyne eirbar frouwe vur eyne houre gegeven were.

Indorsat: Attestata contra Michaelem super cognicione sue vitrice.

Vgl, das Protokoll von 1571, Mai 7.

\section{3.}

\section{Verhandlung gegen Wilhelm von Aachen wegen Unzucht mit seiner Magd und Ehebruch. [1489-1492. $]^{1}$ )}

Dit is alsulchen kuntschaft, as gehoirt is up sulchen ebruch, as Wilhelm van Aiche by synre huyssfrauwen mit Girtgin van Essen, die syne mayt zo syn plach, begangen hait. Darup dat die parthyen herna geschreven ... getzuicht und gekundt, inmaissen herna foulgt....

AuBer verschiedenen für den Fall belastenden Zeugen tritt Johann von Randenroede auf und berichtet von weiteren Ehebrüchen Wilhelms, desgl. Konrad v. R.

Wilhelm van Randenroede sait, yeme sy kundich, dat hey waill zo anderen tzyden mit Wilhelm van Aichen van Antwerpen komen sy umbtrynt dry uyren na myddage bynnen die stat Coelne. Ind do have Wilhelm in gebeden ind yeme gesacht, dat hey mit yeme gayn weulde up eyn heymlich ende, alda weulden sy noch froelichen syn. Do sy hey Wilhelm gefoulgt, und Wilhelm hait in geleyt in eyn bierhuyss, aldair ouch tzwae frouwenpersonen komen syn. Mit denen haven sy alda gesessen, gessen, gedroncken ind froelich geweist, bis dat it gantz duyster ind avent worden was. Umbtrynt tzien uyren in den avent, do sy Wilhelm mit yeme uyss deme bierehuyse gegangen bis up sent Apostelen straissen ind have alda eyn huyss upgeslossen, darinne die vurschreven Gyrtgen van Essen woinafftich was, ind hait die selve mit eyme anderen slayffende befunden ind sy deshalven sere tzornich geworden up die selve Girtgen, sagende: "Got geve der huyren eynen droess ind ovell. Ich hayn yre bedde ind podde

1) Datiert nach den Turmmeistern. 
gegeven ind sust vast anders, ind hedde in vill gestanden. Dat weulde hey allet weder hoelen, umb dat die selve Girtgyn yeme die untruwe gedain hedde “...

Indorsat: Attestata contra Wilhelmum de Aquis super adulterio.

4.

\section{Verhandlung gegen den Taschenmacher Wolfgang van Ween wegen Notzïchtigung seiner Magd. 1504, Sept. 17.}

In yntghainwerdichiet der eyrsamer heren Thoenys van Lomersshem ind Goysswyn Woulff, thoyrnmeystere, ind Johan DuyBbergh, vleyschmartmeyster, alss geschyckde heren und frunde zerzyt unsser heren v. r. synt diesse naegeschreven gefraycht by yren eyden ind freuwelichen truwen de warhiet zo sagen, wat yn wysslich ind kundich yst, den handell beruerende tuschen Woulffganck van Ween, teschmecher, ind eynre frauwenperschoenen genant Barbar Hoenenackerss, Clayss Borden doychter, de desselven Woulffgancks mayt yst gewest, ind der selve Woulffganck de gemelte Barbar, sin mayt, mit kynde gemacht hayt by synre eliger huysfrauwen.

Item herup de gemellte Barbar geclaicht ind gesacht hayt, wie sy by deme selven W. t. eyn zyt lanck gewoent have, ind desselven W. huysfrauwe up eyn zyt zo Nuysse wass gezogen, so dede yr der selve $W$. so vyll leydz an mit plucken ind dynssen ${ }^{1}$ ) ind hette gerne synen wyllen myt yr gedain ind woulde yr yn der nacht nae up yr kamer. Doch en schaffde hey synen wyllen up de zyt nyet myt yr, want sy is yeme nyet gestaden woullde, wass ouch up de zyt nyet dan 15 jayr allt. Dan darnae oever 4 wechen dede yr der selve $\mathrm{W}$. weder so vyll leydz an, dat hey synen wyllen van yr kreych....

\section{5.}

\section{Verhandlung wegen geschlechtlichen Unfugs mit einer Wahn-} sinnigen. 1505, Aug. 22.

Der Inhalt des hier mancher Einzelheiten wegen fortgelassenen Protokolles geht aus dem Vermerk (Indorsat) hervor: Dicta testium in causa puelle tonse in vericundia.

1) Plucken (nhd. pflücken) = zausen. Dynssen = schleppen. 
6.

\section{Bestrafung Juttas vor S. Agatha wegen schwerer Kuppelei.} 1517, Mai 24.

Liber Malefactorum sub J. (G. 204).

Jutta Keuffersse vur Sant Agathen hait hinder unsen herren gefencklich gesessen, umb dat sy mit kuppelye umbgegangen und vill gebruycht, ouch eyn iunck maetgin van 20 jaren mit eyme andern manne up yre eygen bett gelacht, der daselffs an yrer syden mit demselven maetgen die nacht gehandelt, da sy selffs by gelegen have. Deshalven sy up den Kax ame Heumart gestanden und darna zur stat uyssgewyst is worden...

Vgl. sub P. (1519, Jan. 27).

\section{7.}

\section{Verhandlungen wegen homosexueller Vergehen. 1484 Juni, Juli.}

a) Beschluß einer Untersuchung darüber (Stein II, $583 \mathrm{f}$.).

Der Pastor von S. Aposteln hat über das Vorkommen widernatürlicher Unzucht geklagt. Auf dem Sterbebette hat ihm einer darüber gebeichtet und auch den genannt, der mit ihm gesündigt hat, ind dat were eyn rych selich man ind hedde wyff ind kyndere, pleige zo raide zo gain ind were eyn mit van den oeversten ... ind wanne hey yem begaende, so verwandelde yem alle syn gebloede... ind so ducke der selve krancke man dem selven rychen burger zo willen geweist were, so hedde hey yeme 1 postulatsgulden gegeven.

Der Pastor kennt noch einen andern Fall und spricht die Befürchtung aus, es seien in Köln im ganzen wohl 200 .

Darauf wird beschlossen, eine Untersuchung anzustellen. Der Pastor von S. Aposteln wiederholt auf nochmaliges Befragen seine frühere Aussage.

Die "doctoiren in der gotheit" erklären sich auf Befragung für gänzliches Verschweigen.

Trotzdem beschließt der Rat, eine Umfrage bei den Pastoren und Pönitentiern zu halten. 
b) Einsetzung von Schickungen (II, 585).

Am 21. Juni werden 8 , am 12. Juli 5 weitere Männer deputiert.

c) Aussagen der Pönitentier und Pfarrer.

Der penitencieir zo den Mynrebroideren hait gesacht, wie hey 3 jaire penitencieir geweist sy ind have bycht gehoirt, so en sy yem des wercks nye vurkomen, dan van den uysswendigen moge yem vast allerleye vurkomen syn; dan doe dat afflais zo sent Johann was, doe sij yem eyn uysswendich alt man zo yem komen ind have yem van den dyngen gebycht.

Der penitencieir van den Prietgeren hait gesacht, id sy leyder wair, dat yem die dyngen vur der zyt, ee hey penitencieir were, wail vurkomen ind seder der zyt, dat hey penitencieir geweist sy, vast vurkomen, ind dat die sunde leyder gemeyne sy, doch me under den armen dan under den rychen. Doch geschie sij ouch van den rychen, as man mit manne.

Der penitencier van den Augustyneren hait gesacht ind ouch slechtlich in allen vraigen darby entlich bleven, hey moge buyssen bychtz in geselschafften ind anders vast allerleye wail hain hoeren sagen; dan wes yem in bychten gesacht sy, des en geboere yem noch intgemeyne noch in besunderheit nyemandtz so sagen.

Der alde penitencieir zo den Frauwenbroideren hait gesacht, hey sy in dat sevende jair penitencieir geweist, yem sy daeby bynnen vast allerleye vurkomen, dar yem en sy van inwendigen sulchen sunden nye vurkomen noch gebycht. Hey en hoffe ouch nyet, dat sulchen sunden bynnen Coelne geschien.

Ein andrer Pönitentier leugnet das Vorkommen solcher Dinge gănzlich.

Der pastoir zo sent Columben hait gesacht, hey en wysse yetzont van den dyngen nyet zo sagen; id en sij yem ouch bynnen 4 off 5 jairen nyet vurkomen, dan daebevoir moechte hey van eyme gehoirt haven, des handels halven zo doin hatte, der were ouch nu lange doit, ind der were nu geurdelt. Sust en wuste hey van den dyngen nyet zo sagen.

Der pastoir zo sent Brigiden hait gesacht: off yem sulchs vurkomen were in bychten off nyet, dat stelte hey zo gode wert; sulchs en geboere yem nyet zo melden. Id were ouch nutzer vers- 
wegen, dan vyll darvan zo sagen. Hey besorge sych aver, dat leyder sulche und der gelychen sachen hudestages gemeyner syn in duytschen landen, dan sy ye gewest syn; vurder en stae jem nyet zo sagen.

Der Pastor zu St. Peter weiß nichts.

Der Pastoir zo sent Mertyn hait gesacht, die dynge, daevan dat men yem gesacht have, die synt leyder me dan waire. Ind id sy leyder darzo komen, as ad Romanos primo geschrieven stae: "masculus cum masculo, femina cum femina" etc. ${ }^{1}$ ) Hey have ouch zo etzlichen zyden die selve sunde mid anderen sunden, die der gelychen synt, frauwpersonen mit frauwepersonen, in syme kyrspele offentlich bestain zo straiffen. So sy hey darumb geschulden, so dat hey have moissen swygen. Dan hey wille sych bes beraiden, ind moechte hey id doin, he weulde so vyll ayntzeichen sagen; men seulde wail nae dairby raiden; hey halde it gentzlich darvur, dat die plaege der Uproiren [1481 - 1482] uys desen ind ander gelychen sunden untstanden ys; hey sachte ouch mit, dair weren brieve geworpen hynder die doeren, die ouch zorissen und verbrant weren, ind dairinne stunden die selve lude genoempt.

Der officiante zo sent Cunibertz hait gesacht: dat hey seulde sagen, dat yem sulchen ind der gelychen nyet vurkomen were, so moeste hey liegen. Id were yem leyder manichwerff vurkomen, doch in vergangenen jairen vur 8 off vur 10 jairen me dan nu mit 10 off 12 , dan bynnen jairs van zwey off dryn, die mit der sunden gehandelt hetten. Id were under woesten, wilden luden ind ouch etzligen anderen, die in gueder naerunge seessen. Hey hedde ouch noch bynnen kurtz gesien, der mit der sunden befleckt were, id were mit der "Veder" ${ }^{2}$ ) herkomen. Doe hedde id yrst bestain zo plantzen. Doch bestunde id sych nu seer zo slyssen.

Darnae hait der pastoir zo sent Mertyn noch gesacht, hey en kunne yn nyet vyll gesagen, dan der Hewmart were desshalven eyn vuyle geselschafft. Hey weulde waile, dat die huysergyn an deme Lynwaedtzmarte ${ }^{3}$ ) affgebrant weren ind dat hey, dessghenen hey darvan jairlichs krygen, untberen seulde...

\footnotetext{
1) Römer 1, 26 ff.

2) Spitzname einer Person.

3) An der Nordwestseite des Heumarkts, vorgelagert vor der Straße "Unter Hutmacher".
} 
Der Pastor von St. Severin hat nur von einem Fall in drunckenschafft gehört. Der Capellan daselbst verweigert mit Hinweis auf das Beichtgeheimnis die Aussage. Der Pastor von St. Abla B weiB nichts.

Indorsat: In hoc convoluto continetur inquisitio ex mandato Senatus facta super peccato muto sive sodomitico, item der herren Theologorum darauff ertheiltes Guettachten.

d) Berichte über Sodomiterei des Joh. Greffroide (hier der Einzelheiten wegen fortgelassen).

e) Bericht über Sodomiterei des Seidfärbers Seger (hier der Einzelheiten wegen fortgelassen).

Vgl. das Protokoll von 1500, Okt. 8.

8.

\section{Verhandlung gegen den Grafen Friedrich von Rietberg ${ }^{1}$ ) wegen} Unzucht mit einer Zehnjährigen. 1516, Mai 23, 24.

In untganwordicheit der wirdiger eirsamer und frommer heren Johanns Smigken, doctor etc., Godartz van Segen und Philips Ropertz geweldrichterenn, Thonis van Glesch und Gierhartz Roiden thormeisteren, Jacob Koufflieffs, Coynraitz van Brenich und Philips [Lücke] as geschickte heren eins woirdigen raitz der stede Coelne haint Heynrich van Bulioin und Celie elude yre dochter Guetgin, die up Vincula Petri nyestkomende yrst zien jair alt wurde, as sy sachtin, gebracht und sich nochmals beclaight, dat die selve yre dochter overmitz den edelen wailgeborn jonckern Fredrichen greven zo Redbergh, doymherre etc., so unerlichen mishandelt worden sy, in allermaissen, wie sy sulchs unsen heren v. r. ingeschreven und klegelichenn zo erkennen gegeven haint, by wilcher clageschrifft sy ouch noch blyvenn, stain und halden, willen, dat man yre dochter darumb fraige und verhore.

So ist die vurg. yre dochter derhalven gefraigt und verhoirt worden und sait dairup, wie dat der upg. juncker van Redbergh up dynxstach, as man unsen heren got zo Sultze droge, ${ }^{2}$ ) nyestledenn up dem Graven zo yr in bywesen Annen yrre moenen ${ }^{3}$ )

\footnotetext{
1) Prov. Westfalen, Kreis Wiedenbrück.

2) Prozession nach Sülz am Pfingstdienstag. (Gïtige Mitt. von Herrn Prof. Wiepen.)

8) Muhme.
} 
gesacht have: "Weist dyner moentgin ${ }^{1}$ ) gehoirsam und doit, wat sy dich heischt doin. Ich will dyr eynen syden harsnoir, eyn pair toffelen unnd schoin geven." So sy sy uyß geheischen und befeilhe yrre moentgen Annen vurs. up den selven dach zo 6 uyren ungeferlich des namiddags zo den jonckern van Redbergh, der sy selffs zuliesse, eynen samelotten lyffrocke und 1 syden wambus an hadde, zu syne wonung gegangen und gesacht: "Joncker, wanne idt uch gelieven und Giertgen vurg. maight sich nyet zornen, would myn moentgen zo uch komenn," hait der obg. joncker sy alleyne, so sy nyemantz mehr alda sach, mit sich hin up eyn camer, dair drye bedden mit glichen schartzen, ${ }^{2}$ ) ein vierkantich disch up 2 schragen ${ }^{8}$ ) stonden und die duyre mit dubbeln slossen waren, by syner maight Giertgen vurs. gefoirt und gesacht: "Will dich dyn moentgen nu haven, so kom her und haill dich." Doe sloess joncker Frederich vurs. die duyre zo und gienck hynwegh, moist sy aldae blyven.

Und gienge mit Geirtgen vurs. up eyn ander camer zo 11 uyren slaiffen. Over ein halff uyre dairna qwam joncker Frederich vurs. us syner camern, dair eyn bedde mit eynem blaen kogeler ${ }^{4}$ ) zo voessen up stoinde, zo yn beiden up yrre cameren komen und lachte sich by sy up dat bedde und gesan ${ }^{5}$ ) yrre; schalte die maigh(t) Giertgen vurs. syn und sachte: "Schambd uch vur got ind die werlt." Zoigh der joncker dickg. synen degen uB und stoich Giertgin damit. Derwyle stege sy aff und kroepe ${ }^{6}$ ) under dem bedde, nam der joncker vilg. sy mit den beynen und warp sy wie eyn frusch weder up dat bedde und sachte: "Wer her kompt, der mois eyn veder hie laissen!" Und gedege ?) avermails an sy und dede yr also wee, dat sy kryschen moiste. Doe sloich der juncker sy und would haven, dat sy swygen sould. So dede joncker Frederich yr so wee, dat sy nyet geswygen konde unnd kreische also lange, dat joncker Frederich zo leste affliesse und lachte sich hynder Giertgen und bleve aldae up dem selven

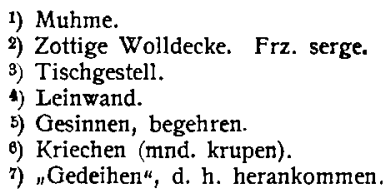


Justus Hashagen.

bedde bis des morgens zo 8 uyren liggen. Do stund hey up und ginge zo deme doym und qwam zo 9 uyren weder und sachte zo yr, dat sy vur dem hilligen cruytze ime doyme zo yrre moengen, die aldae were, genge. Fuegdenn sy sich van stunt dae hyn. Und so sy yr moengen aldae nyet en fant, sy sy [zo] yren alderen heym gegangen.

Eodem anno Saturni 24 Maji.

In presencia prescr. dominorum beider geweldtrichteren, thornmeisteren und Jacobs Koufflieffs synt Metzgen van Overroide und Tryngin Strichers, beide hevelsche, ${ }^{1}$ ) des obeng. handels halven gefraigt und verhoirt worden und sagen dairup, wie sy Grietgen, Heynrichs Buljoins dochter, besichticht, und also befonden worden, dat sy an yre freuwelicheit mishandelt geweist und waill zo sien was, dat eyn mansmynsche mit yr gehandelt und gnoich, umb syn boißheit zo volbrengen, gearbeit hadde. Dan wer der selve geweist, sy yn nyet kundich. Und dat selve metgin were zo deme handell unbeqweme. ${ }^{2}$ ) Indorsat: Kuntschafft tuschen deme junckeren vane Retberge und Henrich Bullions dochter. Beilage: Rietberg an das Domstift, Bonn 1516, Juli 9.

Dazu Liber Malefactorum sub E 1516, Nov. 21.:

Engyn lynwyrckersse [oben Anna genannt] hait hinder unsen herren in gefencknisse gesessen, umb dat sy eyn cleyn maetgin van 11 (!) jaren, dat by yr woynde und Heinrich Bullion kufferslegers dochter geweist ist, upsetzlich by iuncker Frederich greve zu Retberch doymherre etc. gesant, der datselve maetgin upgeslossen und die nacht moitwillichlich mit yem gehandelt soulde haven. Die dan na eyner guder zyt, die sy hynder unsen heren gesessen hait, desselven gefencknisse gnedencklich erlaissen ist....

9.

Verhandlung gegen den Goldschmied Heinrich Brenich wegen Unzucht mit einer Fünfjährigen. 1524, Jan.-1528, Jan.

(Turmbuch I, B1. 44a-48b.)

... Up clage und supplication in berichtzwyse Heinrich Hannemans huysfratwen etliches handels halven, Heinrich Brenich goltsmit mit irer dochterlyn van vunff jaren begangen sol haven,

1) Hebamme.

2) Unfähig. 
ist gemelter Brenich gefencklich angenomen und hinder unse heren zo thorne komen.

Inhalt der supplication volgt hernach alsus luydende:

Gnedigen, lieven heren, so dan ur genaden mir haint doin bevelen die sache, so wie sych H. B. tghain mynen huysswirt und mych begangen hait, wil ich ur genaden nit verhalden, dat myn huysswirt und ich mit zweyen unsen kinderen up sent Steffains dach niestleden uss dem doim under predic heymgegangen. Und so myn huysswirt vur was und by der junfferen zom Hirtz stande und ich na vurgemelten Brenichs huyss gekomen, hait hey mit mehe anderen vur der duerren gesessen. Und as hey mych sach, is hey upgestanden und oeffentlichen gesacht: "Enge locher synt gut wijt ze machen, as etzliche lude willen, und sulde sy die pestilentz erwurghen, “ byn ich stillswygende vurby gegangen.

Und so myn huysswirt und ich nu heym gekomen waren, ist gemelter Brenich in synem paurrock vur unse duer up die strasse komen und mych schentlichen und uneirlichen versprochen und ein meer ${ }^{1}$ ) gescholden und gesacht: "Du verschems dyn kynt." Dairup ich ime antwort gaff, als sy dat kind gestorven, so sy dannoch syn boeBheit niet doit; also dat ein groisse menichte von folck alda vergadert ${ }^{2}$ ) was, ist gemelter Brenich damit nit gesedicht gewest, sonder denselven avent umbtrent 6 uren, as die frauwe zum Birboum tghain mir oever und ich noch vur der durren stoenden, weder vur uns huyss mit eyme gesellen komen, eynen langen degen uff syner syden gehatt und den mit der hant angefast und zo mir komen, so dat die frauwe zom Birboum mych int huyss stiess und die duer zuslossen, rieff Brenich oeffentlich: "Du boesswicht, kom heruss, bistu koen! Und hedde ich den boesswicht, ich wolde mynen hals an yn strecken." Dairup myn huysswirt und ich allit gefragen.

Damit nu u. g. grunt der sachen verstain moegen, geven ich u. g. (nit clagwyse, ouch Brenichs bloitz noch hoge straiffe nit begerende) ${ }^{3}$ ) sunder as ein bedruckte moder, die yrs kindes mitlyden vurmals gedragen, und wie ich dat erfaren hain, mitlydlich und underthenlich zo kennen, und ist also: Als gemelter

\footnotetext{
1) Mähre.

2) Versamneln

3) Die Klammer in der Hs.
} 
Justus Hashagen.

Brenich by mynem huysswirt 10 jair gewoint haite und unse dener geweist, hait hey mit mynem kinde, dat umbtrent vunff jair alt was, fruntlichen gesprochen und yem allemails eynen appel oder ettwas kruytz off koechen gegeven, also dat dat kynt as eyn kynt gehuychnisse $^{1}$ ) zo ime drach. Hadde ich waill gehoirt, dat hey sachte zo dem kynde: „Barbelgen, du moiss eyns in uns huyss und woinde do by Coisfelt - komen, da synt noch frische jonffergen, die mit dir spiellen ", hain ich dairup gheyn acht gehatt. So sal gemelter Brenich eyns up eynen Sondach under der vesper, des disen somer vunff jar wirt, dat kynt genomen und heym in Coisfeltz huyss geleit, boven up eyn kamer, genant die Kirchoffs camer, alda neder gelacht und synen willen (wie wail dat kynt sere schrie) ${ }^{2}$ ) mit yem gehadt und na der handlung ime eynen appel gegeven und daby verboden, mir als eyner moder nit zo sagen, anders wurde ich idt doitslain. Und so hait sich dat kynt dairna geclagt unden an syme lyve; und so ich dat besach, meynte ich, id were sent Quiryns gnade ${ }^{8}$ ) geweist, und det kynt mit dem wasser geweschen und mit salven geheilt mit grossen smertzen und ellende. Doch hait id van der zyt an syn wasser nit moigen halden.

Dairna ist gemelter Brenich van Engelbert Coisvelt goldsmit gefaren und by Peter van Zulp goldsmit komen wonen, hait genanter Brenich avermails mit guden worden mit sych gelungert, ${ }^{4}$ ) up ein camer gefoirt, up ein kussen gelacht und avermails synen willen und unvuegt, wie zovoeren, mit yem begangen, als dat kynt sulchs weder mych bekant hat. Dairna umbrent dry ferdels jars sal gemelter Brenich, as man up der goltsmede gaffel sent Loyen ${ }^{8}$ ) broderschafft dienst ${ }^{6}$ ) gehalden hait, dat trummen und pyffen da waren und myn kynt mit anderen kynderen zo dem spill geloufen, odir villicht uss mynem huyse gehoilt und dat kynt ein trap aff und ein trap up geleit, up ein kamer, dair ein bedde stoinde, gelacht, die camer zogedain und avermals synen willen mit dem kynde gedain.... ${ }^{7}$ ) Sulchs hait dat kynt eirst unserem

\footnotetext{
1) Erinnerung. Vgl. "minne".

2) Die Klammer in der Hs.

9) Eine Seuche.

4) Im modernen Nd. als absolutes Verb fïr "betteln" gebraucht. Hier "locken“.

5) Eligius.

6) Zunftessen.

7) Der weggelassene Passus zeigt die Frivolität in wahrhaft erschreckender Weise.
} 
diener Jacob, dem got gnade, gesacht, der mych dairvur warnde; und so ich dat hoirte, bedacht ich mych des kyntz gebrech, und van stunt an dat kynt by mych genomen und gefragt; haid id mir alle diesen handel, wie vur erkliert steit, ertzalt und gesacht, wie Brenich mit ime gehandelt hette. Hain ich also genantem Brenich van stunt an by myner magt boden gesant und ime sulchs in bywesen des kyndtz alle bekentnisse des kyntz, wie vurschreven is, under ougen gehalden. Und so ich in der sachen haestich kalde, badt hey mych, dat ich hoefflich spraiche und ine nit verschemde, dat des dat gesynde nit gewair wurde; antworde ich, myn gesynde wiste dat besser, dan ich. Und zolest badt hey mych umb gotz willen, dat ich gheine worden daevan machte und mynem manne ouch nit sagen wulde, dat ich dan mit bedrucktem hertzen umb myns kyndtz ere gehalden und gedult gehait. Doch ist gemeyner naberschafft da van wail bewust. Und so hey nu syn huysfrauwe haven solde, hain ich sy guder meynonge dairvur gewarnt und mit Heinrico Herck und syner huysfrauwen, ouch Johan Poilhem und Adam goldsmitz huysfrauwen untleden ${ }^{1}$ ) und ime ouch sulchs gesacht, dat hey dairby gelaissen hait.

Und so nu dat kynt und Jacob vurs. beide doit synt und wir dat kynt mit krentzen haint laissen begraven, so id noch nit mehe dan nuyn jair alt was, koempt gemelter Brenich koenlich hervur und vermeynt die sache zo verdadingen ${ }^{2}$ ) und mych zo beschemen, als solde ich die dingen oever yn gelogen haven, dat ein yeder from hertz ermessen kan, ghein moder doin sulde. Ouch, g. 1. h., dwyle myn kynt vierdehalff jair geleifft und zo vil malen in syme leven langs Brenichs huyss so in hilgen drachten mit groenen krentzen up syne hoeffde ${ }^{8}$ ) gehatt und ime hare gegangen, und Brenich des gheyne bekroenunge ${ }^{4}$ ) noch eynige worde dairvan doerren gewagen noch bekroent, dan nu id gestorven und mit krentzen begraven ist, mit der suster untboden, hey woille sulchs verantworden, und vermist sich voiss by voiss zo setzen. Und hoffen nit, dat sich sulchs in diesen falle gebueren sulle, sunder

\footnotetext{
1) Verstorben.

2) Verteidigen (mnd. vordegedingen).

3) Haupt. gehen dürfen.

4) Klage. Nach Verlust der Jungfräulichkeit hätte das Mädchen nicht mit Kränzen
} 
dat mir ind mynem armen kynde geschiet, moiss ich duldichlichen lyden.

U. g. getruwe burgersse

Cathringen Hannemans.

H. B. leugnet alles ab (45). Dann folgen die Zeugenverhöre $(45 b-48 a)$. Die ersten 8 , die sich auf unwesentliche Punkte beziehen $(45 b-46 b)$, sind fortgelassen.

(46b) Giertgin wilne Sweders vassbenders dochter sall kundich syn, wie Barbelgen des wardeins dochter yr allen gebrech und handel wie vurs., dairzo dat gebrech syns lyffs geclagt have. Antwort Guetgin vurs. by irer junfferschafft, wie dit kynt eyn mal in yrs vaders huyss in der stoeffen have gesessen und zo Guetgin gesprochen: „Guetgin, weis du nit, warumb ich Brenich nit moiss ansprechen?" Have sy gesagt: „Neyn“. Hat Barbelgen gesprochen: „Ich will dirt sagen. Hey hait mych up ein mail up der gaffelen up ein kamer geleit und.... Vort gehandelt, als zo mercken steyt. Sagt vort: wanneir dat kynt Brenich sach, have id sich gentzlich untsatzt, als sy zo vil malen gemerckt have... ${ }^{1}$ )

(47b) Thoenis steynmetzer zuygt, wie hey gerucht boven Marportzen vur Hannemans huyss gehoirt, dat Brenich die frauwe ein meer gescholden, have die frauwe weder gesprochen: "Weerstu nye oever mynen durpel komen, dat sulde zweyhundert gulden wert geweist syn." Do have der wardeyn ouch zo Brenich gesacht: „Du haist wail so vil gedain, dat man dych an armen und beynen [an] perde spannen sulde und ryssen dych van einander...

Dit nageschreven is ein kuntschafft under der stat siegel van Nederwesel durch Heinrich Hanneman erlangt und ingebracht. [1528, Jan. 20.]

Ich, Gerit Bungart, burgermeister der stat Nederwesel, doin kunt und bekennen in diesen openen brieve, dat vur my als burgermeister derselver stat Wesel komen und erschenen is Heesskin van NuyB und hefft uth dwange des rechten und uth versoecken Heydenrich Hanneman, ingeseten burger der stede Colne, mit oeren lyfflicken fyngeren gestaefftz eydes, oer rechter hant up oer luchter ${ }^{2}$ ) burst legende, lyfflicken tot gode und den heilgen gesworen und behalden, dat oer wittich und kundich is,

3) Hier und weiterhin sind 13 nebensächlichere Zengenaussagen fortgelassen.

2) Links. 
dat sy vur ein dienstmagt gedient hefft bynnen der stede Coelne by dem genanten Heinrichen Hanneman boiven der Mairportzen und in denselven huyss dan desselven Heinrich dochter, geheeten Barber, die wilcker noch nit alder dan tuschen vunff und seess jaren tertyt was, van Heinrich Brenich, syn knecht twesen plach, geleit is in Engelbert van Koisfeltz huyss goldsmit syns meisters tertyt up die Kerkhoffscamer und synen unreynen willen mit oer int eirst aldair gedain hefft und oer do eynen groten appel gegeven, siggende tot oer: "By lyve segt dat dyner moder nyet! Off sy sleit dy doit." Und wo dat kynt vurs. so grot gebreck dairna an den tweden und derden dach und vont dairna an syn heymlicheit, dat man last und lyden dairmede gehat hefft, bis tortyt sy und oer frauwe datselve weder geplaestert und geheilt hebben. Dairna is dieselve Heinrich Brenich van dem bemelten Engelbert gefaren und qwam by eynen to wonen, geheiten Peter van Zulpen goltsmit, hefft hey dat kynt vurs. up ein tyt weder gekregen und up ein kamer geleit und up kissen gelacht und glychmatich wie vurs. mit oem gehandelt. Then derden hefft Heinrich vurs. des anderen dages na sent Loyen dage dat kemelte kynt uth syns vaders huyss gehailt und upper Gaffelen, dair die goldsmidt gesellen by yn waren, und ein trap vam sale affgeleit und ein trepkin weder up ein camer, dairinnen ein bedde stoinde, und synen onreynen willen mit oem aldair ock begangen, allit als dit unmundige kynt tghen der egenanten Heesken selffs muntlich bekant und gegiet hefft. Und as dat geschiet und die undait vurs. uthgebroken is, hedde die moder des kyndtz Heessken an denselven H. B. gesant, omme by er to komen. Und as hey in oere stoiven bynnen oeren huyse gekomen is, hefft sy oem alsulchen undoigt und boese geschefften, mit oerem kynde begangen, mit harden worden vurgehalden, hefft $\mathrm{H}$. oer under vil anderen worden gebeden, oem niet to verschemen. Wulde sy sulx oin synen willen niet doin, so sulde sy oer selffs kynt nit beschamen und allet sunder argelist.

Und want dan allet wie vurs. vur my G. B. als burgermeister getuygt und vur rechten gedragen is, des in urkunde und tuge der wairheit heb ick der stat secreit segel ad causas up spacium desselven placcatz gedruckt. 
Gegeven in den jairen unss herren duysent vyffhundert acht und twintich, maindays Fabiani et Sebastiani martirum.

10.

\section{Verhandlung gegen Sybe Wetschemecher wegen Unzucht mit} einer Minderjährigen. 1527, März 28.

(Turmbuch I, Bl. 51b-52b.)

... Sybe Wetschemecher ist gefencklich angenomen worden, umb dat hey mit eyme sere jongen maetgin ungebuerlich und undoigentlich gehandelt sulde haven. Derhalven hey anno 1527 den 28. dach Martii durch die eirsame heren Heinrich Unverdorven, Thys van Poilhem, Franck van der Ketten und Laurens Swartzenberg as verordente eyns eirsamen raitz der stede Colne examineirt und verhoirt ist worden.

Zom eirsten hait dat maetgin genant Gertgin sich beclagt, wie S. vurs. up ein zyt zu yr komen und gebeden have ime zo vergunnen, sy anzotasten, wie ein maetgin, genant Amelungk, van dem alden manne Heinrich Kremer angetast wirt, dat sy ime geweygert und affgeslagen have. Dairna have id sich begeven, dat Gertgin vurs. up ein zyt up dem heymlichen gemach geweist und vort up ire kamer gegangen. Sy S. gekomen und have die duer zo gedain und syn metz boven die klynck gestechen und sy geweltlichen angegriffen, up die kussen geworpen... und also synen willen mit $\mathrm{yr}$ gedain, in maissen frauwen und man zosamen zo doin pligen. Und als sulchs geschiet, have Gertgen zo ime gesprochen mit schryenden ougen: „Is dit angetast, dat wil ich myner moder sagen!“ Have yr S. geantwort: „Wa du dit dyner moder sagist, so wil ich sagen, du haiff's mych dairumb gebeden. Wiltu aver swygen, so wil ich dir ein schryngin ${ }^{1}$ ) geven, dat ich dir geloifft hain." Diese handelunge have Gertgin Annen, irer moders maigt, geclagt durch gebrech und wehes halven. Die dan dat der moder vort verkundigt hait. Und also vur den dach komen.

Uff sulchen clage Gretgins vurg. ist egenante S. vurgehalden und syn verantwerung dargegen gehoirt:

Zom ersten ine gefragt, off hey ouch eyniche geloeffte zo

1) Schrein, Kästchen. 
Girtgin Thoenis dochter van Unna vermesse, geantwort, sunderlich niet, dan uff ein mail eynen koichen in syner hant gehadt und gesprochen have: „Den wil myner zokomender huysfrauwen geven." Den dan Gertgin sunder underlais begert und na (!) sich genomen have.

Vorder sagt S. vurs., wie hey dry jaire by Thoenis van Unna gewoint have und van der dienstmagt, genant Hylgin, gehoirt, wie Gertgin die dochter zo vil malen mit eyme alden manne, Heinrich Teschmecher genant, zo schaffen gehatt; so have hey up ein mail mit Giertgin vur yrs vader duerre gestanden und Geirtgin in den bosem getast. Have sy sych geweygert, have hey zo ir gesprochen: "Sach, weygerstu dych myner? Warummb weigerstu dych nyet an dem alden man, der so dick mit dir zo doin gehat und manich mail so und so gedain hait?" Have Geirtgin geantwort: "Ja, dat were lange geschiet; dat hette sy gebycht. Wanne hey yr ouch etwas geven wulde, wie der alde man gedain, so wulde sy id yem ouch gehenghen. “1) Have hey gefragt: "Wat sal ich dir geven?" Sulde Geirtgin geantwort haven: "Giff mir ein schringin, wie Amelunck hefft, so wil ich id doin." Asdo sulde S. gesprochen haven: "Wan dyn moder sulx gewair werde, wie du daran komen weirst, wat wuldestu dan sagen?" "Dat is wair", sulde Geirtgin gesprochen haven, "so giff mir almails vur dat schryngin moergere und naelden, ${ }^{2}$ ) dat moder nit gewair werde“. Damit samender hant up die leuven ${ }^{3}$ ) gegangen, synen willen mit Geirtgin vurs. gehandelt heve, wie man und frauwen zosamen handelen.

Folgen weitere Zeugenaussagen.

Und als obgemelter Sybe umbtrint 6 off 8 mainde zo thorne gesessen hait, ist hey der gefencknisse oevermitz synen gewoenlichen urfreden gnedencklich erledigt worden.

11.

Verhandlung gegen Jorge von Attendorn, genannt Walthase, wegen Unzucht mit einer Siebenjährigen. 1528, Jan. 15, Febr. 24. (Turmbuch I, Bl. 49a-b.) (Hier fortgelassen.)
1) Erlauben.
2) Nadel.
3) ObergeschoB, Speicher. 